\title{
Output Power Estimation of High Concentrator Photovoltaic Using Radial Basis Function Neural Network
}

\author{
Mensah K. Anaty 1,2, 3, Yaser I. Alamin ${ }^{3}$, Khalid Bouziane ${ }^{1 * *}$, Manuel Pérez García ${ }^{3}$, Reda Yaagoubi ${ }^{4}$, \\ José Domingo Álvarez Hervás ${ }^{3}$, Merouan Belkasmi ${ }^{1}$, Mohammed Aggour ${ }^{2}$ \\ ${ }^{1}$ Université Intenationale de Rabat, LERMA, School of Renewable Energies and Petroleum Studies, Technopolis, 11000 - \\ Sala el Jadida, Morocco \\ ${ }^{2}$ Université Ibn Tofail, Faculty of Science, Renewable Energy and Environment Laboratory, 14 000- Kenitra, Morocco \\ ${ }^{3}$ Universidad de Almería, CIESOL Research Center on Solar Energy , Agrifood Campus of International Excellence, \\ ceiA3E-04120 Almería, Spain \\ ${ }^{4}$ School of Geomatics and Surveying Engineering, Hassan II Agronomic and Veterinary Institute, Rabat, Morocco \\ *Corresponding author: khalid.bouziane@uir.ac.ma
}

\begin{abstract}
High Concentrator PhotoVoltaic (HCPV) is a recent PV technology generating electricity from solar radiation. Unlike conventional PV systems, it uses lenses and curved mirrors to focus solar rays onto small, but highly efficient Multi-junction (MJ) solar cells. Solar tracker and cooling systems are part of a standard CPV facility. Due to the complex design of an HCPV system, the output power estimation becomes a very hard task. In contrast, Machine Learning (ML) methods, and more specifically Artificial Neural Networks (ANNs), provide very suitable solutions for modelling complicated systems. The aim of this work is to develop a Radial Basis Function Neural Network (RBFNN) model to predict the output power of an HCPV facility. RBFNNs have a simple topological structure and their ability to reveal how learning proceeds in an explicit manner. Our results showed that the RBFNN model provides more accurate estimation of output power compared to the ASTM-E2527 based on the same dataset.
\end{abstract}

Keywords- Power Estimation, High Concentrator PhotoVoltaic, Machine Learning, Radial Basis Function, Neural Network.

\section{INTRODUCTION}

In recent years, the fact to introduce a clean energy as alternative sources, such as solar energy, is expected to reduce the $\mathrm{CO}_{2}$ emissions.

Photovoltaics represent the most popular solar energy system and more specifically, Concentrator PhotoVoltaic (CPV) is one of the most promising renewable energy technology able to reduce the fossil fuel dependence [1]. CPV uses cheap optical devices like lenses and curved mirrors to focus sunstrokes onto small, but highly efficient Multi-junction (MJ) solar cells. Solar tracker and cooling systems are part of a standard CPV facility [2].

In both, development and the market integration of every energy production system, energy prediction represents a key factor. Unfortunately, due to the design complexity of a CPV system, the output power estimation is very difficult and complex task. Some methods for output power estimation of a CPV module had been elaborated by earlier CPV researchers. However, the implementation of most of these methods are laborious because of several reasons such as: 1) the need of some intrinsic parameters of the MJ solar cell used in the CPV assembly and 2) the requirement of complex, accurate and sometimes highly expensive devices for specific tests [2].

In contrast, Machine Learning (ML) methods, and more specifically Artificial Neural Networks (ANNs), are used to identify the system dynamics without explicitly knowing the interactions between its components [3]. ANNs are currently used for the modeling of solar power and energy systems in a wide range applications both in the demand side [4] and the production ones [5, 6]. Some researchers [7] had developed a feed-forward neural network to estimate the maximum power of an HCPV module located in the south of Spain, using Direct Normal Irradiance (DNI), Air Mass (AM), Precipitable Water (PW), Air Temperature (Tair) and Wind Speed (Ws) as inputs. This network was trained based on Levenberg-Marquart back-propagation algorithm, which finds only local minimum. It should be noted that ANNs have a lot of different uses outside the interest of this work.

Choosing ANNs in this work is justified by the configuration of the plant. Among other sources of uncertainty and no linearity, three strings on the same plant imply that the value of the DNI impinging on the multi-junction solar cells is not the same. This makes, together to the rest unknown variables and processes, a complex task to predict the output power of the system. In this work, an RBFNN model has been developed for the prediction of the maximum power of a CPV system located on the campus of International University of Rabat (UIR), in the middle-West of Morocco. In addition, the model of the Standard ASTM E2527, which is one of the simplest mathematical models used in the power rating of CPV systems, has also been implemented. The ASTM E-2527 model takes into account atmospheric parameters such as (DNI), air temperature, and wind speed. Its results were compared to RBFNN results. 


\section{HPCV SYSTEM}

This study has been conducted on a whole HCPV system consist of $108 \mathrm{CPV}$ modules mounted in three strings of 36 modules and connected in series. The CPV facility is located on the campus of International University of Rabat (UIR) in the middle-West of Morocco (geographical coordinates: latitude $33.982^{\circ} \mathrm{N}$, longitude $6.7248^{\circ} \mathrm{W}$ ). Each $\mathrm{CPV}$ assembly is made of a lattice-matched GaInP/GaInAs/Ge MJ solar cell, a PMMA Fresnel lens as a primary optic, and a refractive truncated pyramid as the secondary optic. The modules have a geometric concentration ratio of 961 and they are built by six cells connected in series. Fig. 1 shows the HCPV plant. Tables I \& II show the technical details of the modules provided by the manufacturer namely Magpower Company. An SHP1 Kipp \& Zonen pyrheliometer has been settled on the solar tracker for the measurement of the DNI.

A weather station nearby located was used to record other environmental parameters, such as wind speed, air temperature, relative humidity and wind direction.

Solar elevation (h) is deduced using a dedicated software installed on the tracker.

TABLE I: CHARACTERISTICS OF THE HIGH CONCENTRATOR PHOTOVOLTAIC MODULES.

\begin{tabular}{c|c}
\hline \multicolumn{2}{c}{ Electrical characteristics } \\
\hline Type of solar cells & $\begin{array}{c}\text { Lattice-matched } \\
\text { GaInP/GaInAs/Ge }\end{array}$ \\
\hline Cell dimension & $1 \mathrm{~cm} \mathrm{x} 1 \mathrm{~cm}$ \\
\hline Concentration & X 800 \\
\hline $\begin{array}{c}\text { Geometrical } \\
\text { concentration }\end{array}$ & X 961 \\
\hline Number of solar cells & Six cells in series \\
\hline Cooling system & Passive \\
\hline Primary Optics & PMMA Fresnel lens \\
\hline $\begin{array}{c}\text { Dimension of Primary } \\
\text { Optics }\end{array}$ & $31 \mathrm{~cm}$ x 31cm \\
\hline Secondary optics & Refractive truncated pyramid \\
\hline Type of cell protection & Bypass diode \\
\hline
\end{tabular}

TABLE II: THE ELECTRICAL CHARACTERISTICS OF HIGH CONCENTRATOR PHOTOVOLTAICS MODULE UNDER STANDARD CONDITIONS CSTC AS PROVIDED BY THE MANUFACTURER

\begin{tabular}{c|c}
\hline \multicolumn{2}{c}{ Electrical characteristics } \\
\hline Maximum power & $110 \mathrm{~W}$ \\
\hline Open-circuit voltage (Voc) & $17,70 \mathrm{~V}$ \\
\hline Short-circuit current (Isc) & $8,65 \mathrm{~A}$ \\
\hline Maximum voltage & $15,27 \mathrm{~V}$ \\
\hline Maximum current & $7,7 \mathrm{~A}$ \\
\hline Temperature coefficients \\
\hline voltage & $-0.3 \mathrm{~V} /{ }^{\circ} \mathrm{C}$ \\
\hline Current & $+8 \mu \mathrm{A} /{ }^{\circ} \mathrm{C}$ \\
\hline Power & $-0.16 \% /{ }^{\circ} \mathrm{C}$ \\
\hline
\end{tabular}

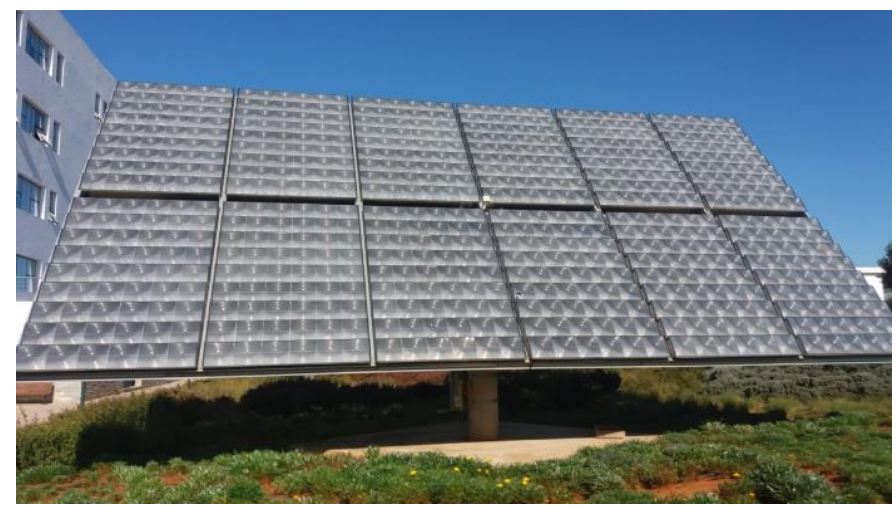

Figure 1. HCPV facility

\section{BACKGROUND}

\section{A. The CPV Power model of the ASTM-E2527}

The model of the standard of the American Society for Testing and Materials exploits a simple and single equation to estimate the maximum power of a CPV module using the DNI, the $\mathrm{T}_{\text {air }}$ $W_{s}[8,9]$.

$$
P_{M P P}=D N I \cdot\left(\lambda_{1}+\lambda_{2} \cdot D N I+\lambda_{3} \cdot T_{a i r}+\lambda_{4} \cdot W_{s}\right.
$$

In the equation above, the coefficients $\lambda_{1}, \lambda_{2}, \lambda_{3}$ and $\lambda_{4}$ are obtained by regression analysis of the HCPV output data. It is noticed that this model does not take into account the spectral effect on multi- junction cells $[8,9,10]$.

Apart from the above-mentioned mathematical model, other existing methods can be found namely: the Lineal model of E.F. Fernández et al [11]; the Sandia National Laboratories Model [12]; the model of Peharz G. et al. [13]; Model of A.J. Rivera et al [14] and YieldOpt [15]. Unfortunately, their implementation is more difficult, compared to the use of the ASTM E-2527 model.

\section{B. Artificial Neural Networks (ANNs)}

Though the above-mentioned methods can approximately model the output power of a CPV system by using linear relations, they do not consider dynamical effects such as changes in irradiance or spectrum, to mention but a few.

ANNs mimic the human brain's biological neural network in the problem-solving process. An ANN can be seen as a black-box that connects the input to the output, with fully connected neurons (nodes), these nodes being connected by weights. They are used for the non-linear mapping between the input data, X, and the output vector, $\mathrm{Y}$, in order to model relations or to detect patterns among them. Less knowable of the system is required in this case. Hence, ANNs could be very useful in complicated systems and models. By using supervised training methods, the parameters (weights and biases) and structure can be determined from data. 
An RBFNN is a three layers feed-forward ANN, an input layer made up of m nodes, a hidden layer with $n$ neurons or RBFs, and an output layer with one node. A radially-symmetric basis function, which can be defined in several ways, is used to activate the $\mathrm{n}$ neurons of the hidden layer. The Gaussian function is the most widely used as activation function:

$$
f_{i}(x)=e^{\left(-\frac{\left\|C_{i}-x\right\|^{2}}{2 \sigma_{i}^{2}}\right)}
$$

In this expression, $C_{i}$ is the centre of basis function, $\|\quad\|$ is the typically Euclidian norm and $\sigma$ is the width (radius).

The output of an RBFNN can be expressed as:

$$
y=\sum_{i=1}^{n} W_{i} f_{i}(x)
$$

Where $W_{i}$ is the weight corresponding to the connection between the $i$ th RBF unit to the output.

While training the network, there are three steps before obtaining the parameters of the RBF units:

i. The unit centers are obtained by K-mean clustering algorithm.

ii. The nearest-neighbor method is used to determine the widths;

iii. By using least square techniques, weights connecting the RBF units and the output units are calculated.

RBFNNs are able to solve nonlinear problems, since unsupervised learning in the hidden layer is combined with supervised learning in the following layer. This makes them suitable for photovoltaic power forecasting.

In this research, the inputs correspond to environmental parameters namely: weather (Tair (Air Temparture), Ws (Wind speed), Wd (Wind direction)), AM (Air mass) based on Solar Elevation (measured or calculated), Azimuth angle of the tracker, DNI and the output power of the HCPV system delayed one time (q-1) and two times (q-2). While in the hidden layer, a wide number of nodes have been tested (from 10 to 20 nodes with one output node which is the output power of the HCPV system).

In general, RBFNN training is performed using a gradientbased algorithm, which minimizes the training error. The training process will be terminated when the minimum of the generalization error is obtained, the error obtained in an unseen data, as training evolves, a spread dataset used for this process called generalization dataset. This scheme is a way to solve the problem known as overtraining. To compare different trained models, with possible different model structure, a third dataset, denoted as a testing dataset, is needed. Hence, three different sets of data are used: i) training, ii) generalization and, iii) testing datasets $[16,17]$. Fig. 2 shows the structure of the ANN proposed in this paper.

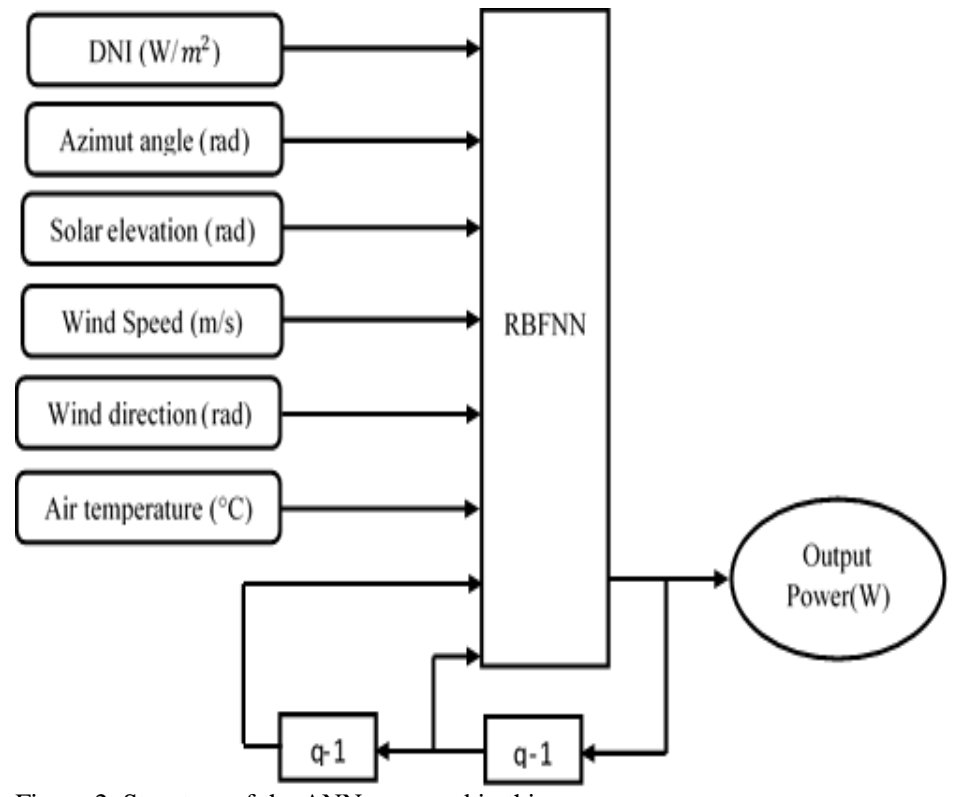

Figure 2. Structure of the ANN proposed in this paper

\section{EXPERIMENTAL SET-UP}

This work takes into account a dataset with data that have been collected during 3 years. More specifically, 92 days have been selected from this dataset. The inputs extracted from different sources have different sampling times $(6 \mathrm{~s}, 15 \mathrm{~s}, 30 \mathrm{~s}$, and 1 min). However, all the data have been resampled to have a sampling time of $1 \mathrm{~min}$ in order to be used in the RBFNN to produce the desired model with a time step of $1 \mathrm{~min}$ and predict up to 15 steps ahead. Fig. 3 shows the scheme of the experimental set-up used in this work.

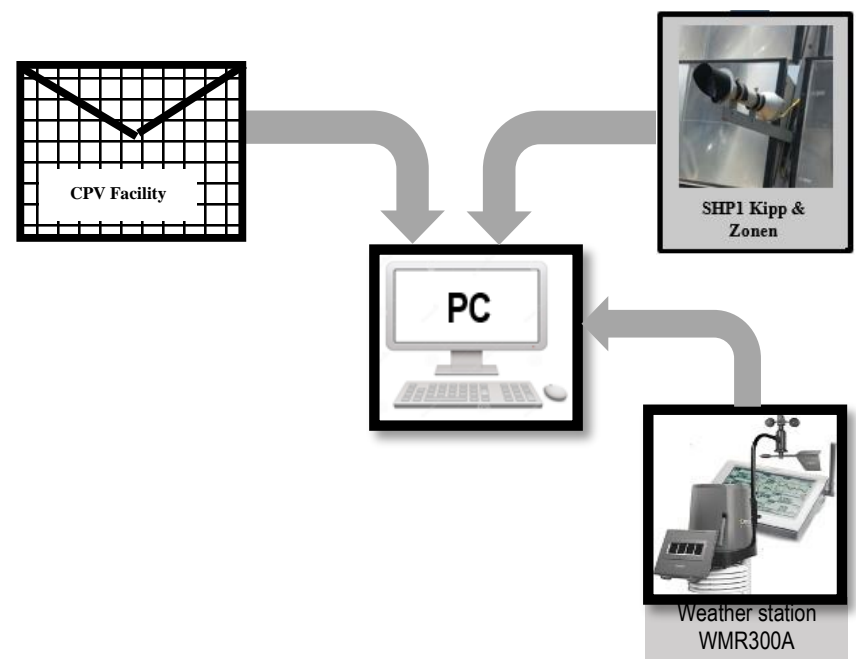

Figure 3. The experimental set-up used to carry out the study at International University of Rabat

The solar elevation can help to calculate approximatively the relative optical Air Mass (AM). AM expresses the ratio of the optical path length of the solar beam through the atmosphere to the optical path through a standard atmosphere at sea level with 
the Sun at apex. It can be expressed as a function of the solar altitude $h$ by [2, 7]:

$$
\mathrm{A} M(h)=\frac{\exp \left(-Z / Z_{h}\right)}{\sinh +0.50572(h+6.07995)^{-1.6364}}
$$

Where, $Z$ is the place elevation $(Z=146 m)$ for the International University of Rabat) and $Z_{h}$ is the scale height of the Rayleigh atmosphere near the Earth surface, equal to $8434.5 \mathrm{~m}$.

The result of the network using AM will be the same if the solar elevation is used because they are two dependent parameters. Hence, the solar elevation is considered as input in our model instead of AM.

\section{RESULTS AND DISCUSSIONS}

In this work, a Radial Basis Function Neural Network had been designed using MATLAB and the same software has been used to extract the coefficients of the ASTM E-2527 model.

The data consists of 92 days from 2016 to 2018. The data was divided into two part of 46 days each (sunny days and cloudy days). Partly cloudy days have been considering as cloudy days, after filtering the data from noise and errors (measurement errors). Three types of data have been created for each dataset; training, generalization and testing. Fig. 4 shows the trend of the output maximum power for sunny days.

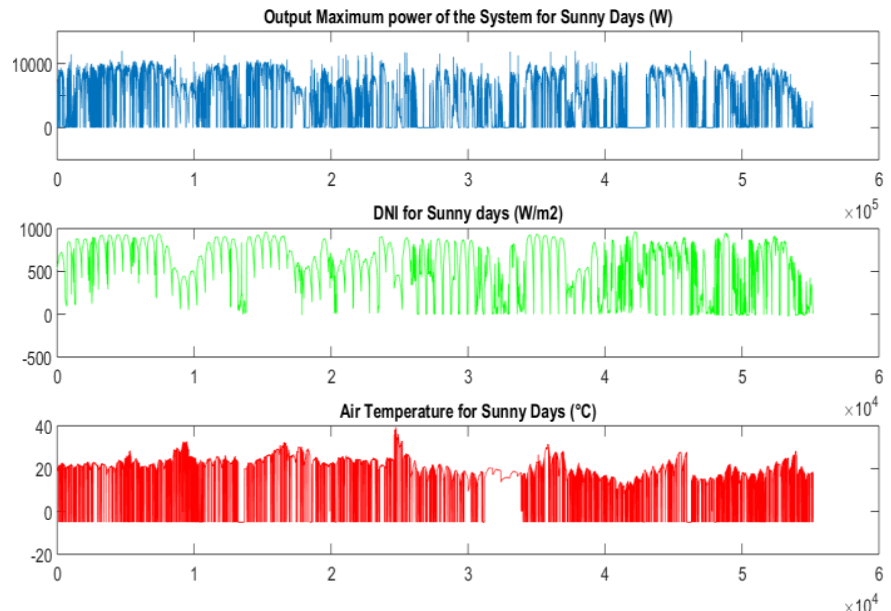

Figure 4. Output Maximum Power and the Corresponding DNI and Tair

The results are presented in two parts:

\section{A. Model of ASTM E-2527}

Table 4 represents the coefficients obtained for the HCPV system according to the ASTM E2527 Model. In table 5, the values of the Root Mean Square Error, between actual and predicted output power for the HCPV system are reported.
TABLE IV: COEFFICIENTS OBTAINED FOR THE MODULE UNDERSTUDY FOR THE ASTM E2527 MODEL

\begin{tabular}{c|c|c|c}
\hline$\lambda_{1}$ & $\lambda_{2}$ & $\lambda_{3}$ & $\lambda_{4}$ \\
\hline 5.1864 & $4.2623 \times 10^{-3}$ & $2.5018 \times 10^{-3}$ & $-1.3254 \times 10^{-3}$ \\
\hline
\end{tabular}

TABLE V: RMSE, $\boldsymbol{R}^{2}$ BETWEEN ACTUAL AND PREDICTED OUTPUT POWER FOR THE HCPV SYSTEM

\begin{tabular}{cc}
\hline RMSE & $R^{2}$ \\
\hline $4.07 \times 10^{3}$ & 0.334 \\
\hline
\end{tabular}

\section{B. RBFNN Model}

For the same data set using DNI from satellite, air temperature, Wind speed, Solar elevation, Wind direction, and $\mathrm{P}(\mathrm{q}-1), \mathrm{P}(\mathrm{q}-$ 2) as input, the RMSE to RMS of the power is $9.2 \%$ which represent $1.3 \times 10^{3}$ watts of the total power. We can easily notice that the RBFNN estimate better the output power than the ASTM model.

The higher RMSE of RFBNN model that is presented in this work is due to some constraints faced in this work, namely:

- The loss in the wires connecting the modules to each other while forming the strings;

- $\quad$ The measured DNI was sometimes not available for all the 92days considered. Since then, we have used DNI from the satellite. The satellite based DNI is less accurate than the one obtained from the pyrheliometer;

- The weather station transmission is sometimes interrupted, making useless the other inputs corresponding to those times.

- The number of days (data samples) are not enough to train efficiently the RBFNN model.

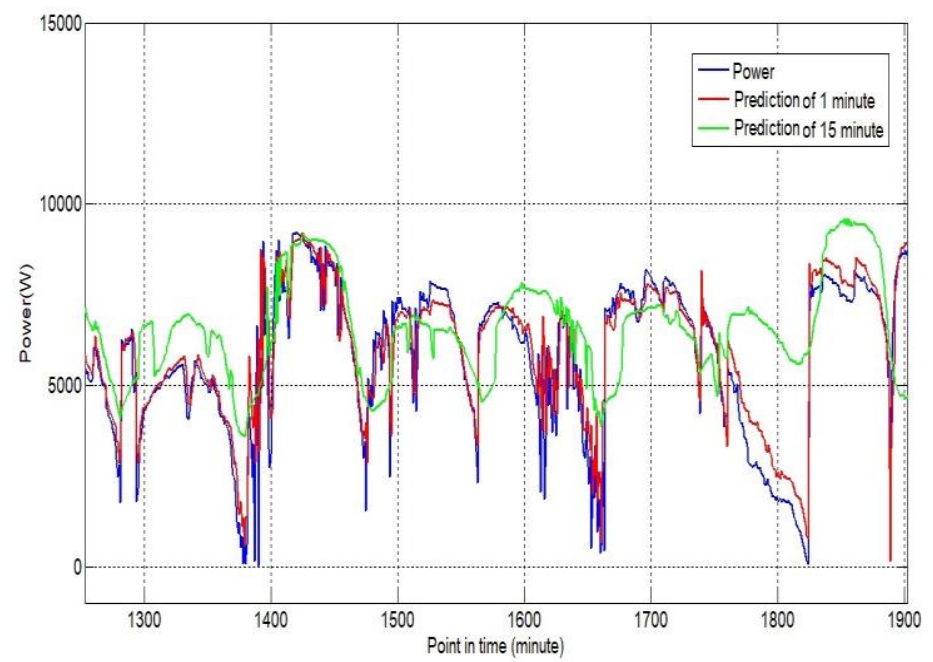

Figure 5. The output power of the system vs the output power of RBFNN model for sunny days 
Figure. 5 shows the power of the HCPV system and the prediction of the RBFNN Model (1 minute and 15 minutes ahead for sunny days). Fig. 6 shows the prediction results for cloudy days.

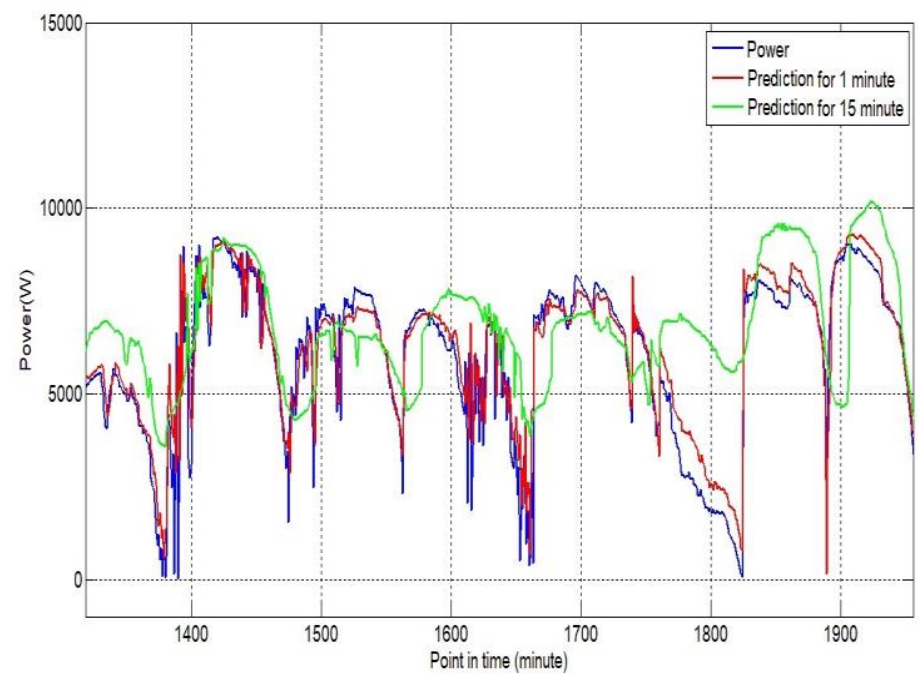

Figure 6. The output power of the system vs the output power of RBFNN model for cloudy days

The weak value of the $\mathrm{R}^{2}$ in the ASTME-2527 is surely due to the same constraints. Moreover, this model has been designed based on measurements on a single module.

\section{CONCLUSION AND FUTURE WORK}

In this work, a Radial Basis Function Neural Network has been designed to estimate the output power of an HCPV facility. The results were compared to those obtained by the ASTM E-2527 model using the same dataset. The accuracy of the models has been evaluated based on the root mean square error (RMSE). The RMSE of the ASTM model is $4.07 \mathrm{kw}$ for the sunny days while RMSE of the RBFNN is $1.3 \mathrm{kw}$, showing that the RBFNN is more accurate than the ASTM model.

In future work, more data will be collected and put into the RBFNN Model to have a better prediction of the system in order to reduce the error values. In addition, other inputs could be added to improve the quality of prediction. Moreover, the use of Deep Neural Networks DNNs (with more hidden layers) in the output power estimation of HCPV will be investigated. Also, more experiments will be carried out with various sampling times and various steps to assess the impact of these modifications on the prediction quality.

\section{ACKNOWLEDGMENT}

This work has been co-funded by IRESEN through its financial support to the projet LOuCOuM and a grant from the Spanish Ministry of Economy and Competitiveness (CHROMAE DPI2017-85007-R). Mensah K. Anaty is an Erasmus+ program internship student and Yaser I. Alamin is a fellow of the MARHABA, an Erasmus Mundus Lot 3 project.

\section{REFERENCES}

[1] R. M. Swanson, "Promise of concentrators," Prog. Photovoltaics Res. Appl., vol. 8, no. 1, pp. 93-111, 2000.

[2] P. Pérez-Higueras and E. F. Fernández Editors, "Green Energy and Technology High Concentrator Photovoltaics Fundamentals, Engineering and Power Plants.” Springer 2015.

[3] A. Mellit, S. A. Kalogirou, L. Hontoria, and S. Shaari, "Artificial intelligence techniques for sizing photovoltaic systems: A review," Renew. Sustain. Energy Rev., vol. 13, no. 2, pp. 406-419, 2009.

[4] Alamin, Y.I., Castilla, M.D.M., Álvarez, J.D. and Ruano, A., 2017. An economic model-Based predictive control to manage the users' thermal comfort in a building. Energies, 10(3), p.321.

[5] Mandel, P., Madhira, S.T.S., Meng, J. and Pineda, R.L., 2012. Forecasting power output of solar photovoltaic system using wavelet transform and artificial intelligence techniques. Procedia Computer Science, 12, pp.332337.

[6] Chen, C., Duan, S., Cai, T. and Liu, B., 2011. Online 24-h solar power forecasting based on weather type classification using artificial neural network. Solar Energy, 85(11), pp.2856-2870.

[7] F. Almonacid, E. F. Fernández, P. Rodrigo, P. J. Pérez-Higueras, and C. Rus-Casas, "Estimating the maximum power of a High Concentrator Photovoltaic (HCPV) module using an Artificial Neural Network," Energy, vol. 53, pp. 165-172, 2013.

[8] ASTM E 2527, "Standard test method for electrical performance of concentrator," American Society of Testing and Materials, 2009.

[9] P. Rodrigo, E. Fernandez, F. Almonacid, and P. Perez-Higueras, "Models for the electrical characterization of high concentration photovoltaic cells and modules: A review," Renew. Sustainable Energy Rev., vol. 26, pp. 752 $760,2013$.

[10] E. Fernández, F. Almonacid, P. Rodrigo and P. Pérez-Higueras, "Model for the prediction of the maximum power of a high concentrator photovoltaic module," Solar Energy, 2013, vol. 97, pp. 12-18.

[11] A. Soria-Moya, F. Almonacid Cruz, E. F. Fernandez, P. Rodrigo, T. K. Mallick, and P. Perez-Higueras, "Performance Analysis of Models for Calculating the Maximum Power of High Concentrator Photovoltaic Modules," IEEE J. Photovoltaics, vol. 5, no. 3, pp. 947-955, 2015.

[12] D. L. King, W. E. Boyson and J. A. Kratochvil, "Sandia National Laboratories. Photovoltaic array performance model SAND2004-3535," Albuquerque, New Mexico, USA, 2004.

[13] G. Peharz, G. Siefer, and A.W. Bett, "A simple method for quantifying spectral impacts on multi-junction solar cells," Solar Energy, vol. 83, 2009, pp. $1588-1598$ 
[14] Rivera AJ, García-Domingo B, del Jesús MJ, Aguilera J. Characterization of concentrating photovoltaic modules by cooperative competitive radial basis function networks. Expert Systems with Applications 2013;40:1599-608.

[15] M. Steiner, G. Siefer, T. Hornung, G. Peharz, A. Bett, "YieldOpt, a model to predict the power output and energy yield for concentrating photovoltaic modules," Progress in Photovoltaics: research and applications, 2014.
[16] Ferreira, P.M. and Ruano, A.E., 2000. Exploiting the separability of linear and nonlinear parameters in radial basis function networks. In Adaptive Systems for Signal Processing, Communications, and Control Symposium 2000. AS-SPCC. The IEEE 2000 (pp. 321-326). IEEE.

[17] Haykin, S. (2005). Neural Networks A comprehensive Foundation. PEARSON Prentice Hall, Ontario. 